\title{
Review of: "Insights and genetic features of extended-spectrum beta-lactamase producing Escherichia coli isolates from two hospitals in Ghana"
}

KENNEDY BOAHEN

Potential competing interests: The author(s) declared that no potential competing interests exist.

The article is very comprehensive and captivating. The style of writing, grammar and diction are on point. In general, the aim of the study design was achieved and the conclusion reflect the aim of the study. I don't have any issues to ask authors to resolve except that the information on supplementary data 1 should be limited to what is needed for this article. 